\title{
On the injury of finger joints in Volleyball
}

\author{
Fanghui $\mathrm{Li}^{1 \mathrm{a}}$, Ying $\mathrm{Li}^{2 \mathrm{~b}}$ \\ ${ }^{1,2}$ Pingxiang University, Jiangxi 337055, China \\ a59558407@qq.com, b359020645@qq.com
}

Key words: volleyball; finger joint; injury

\begin{abstract}
This research through the questionnaire survey method, interview method, questionnaire survey was conducted in Pingxiang Institute of public sports 600 students, to understand students cause joint damage mainly lies in that learning in volleyball, volleyball extracurricular activities: lack of preparation, professional technical movement is not correct, incorrect judgement of the ball; and put forward the corresponding preventive measures according to the reasons, in order to reduce the finger joint damage. The purpose of this study is to meet the school physical education teaching and improve the quality of volleyball teaching, to provide a reference for reducing the damage.
\end{abstract}

Volleyball in the United States came out, by the people of all walks of life in the world has been the rapid popularization and promotion. Especially in the national normal universities have set up the volleyball elective course, by modern college students; volleyball technology is relatively simple, easy to use, students can set up separate practice, practice, practice a pass on a pad; pad can also pass the smash group entertainment; but in the teaching or practice process, it is prone to finger joint damage. Therefore, it is very valuable to understand the injury of Volleyball in students.

\section{An investigation of the object and the situation analysis}

1.Pingxiang Institute of the survey 2014, class 2015 public sports volleyball option class students, the content of the survey for the volleyball in the middle of the joint injury. The complete statistics of sports injury investigation, questionnaires, students, teachers to supervise students to fill the questionnaire, volleyball refers to the joint damage in the investigation of CPC issued 600 questionnaire for students of grade 14 in 300, 15 grade 300. According to the survey, the rate of injury of the joint was 35.5 and 2015, and the rate of injury was 32.5\%. 2014

2.Refers to the joint structure relative to other joints of human body is very simple, a joint is the most neglected, is the basic of trochlear, except the thumb proximal joint, can only do flexion and extension, the dorsal capsule has a little relaxation, three ligament reinforcement. Therefore the magnitude of limited flexion will produce damage, damage in the form generally has two kinds: one kind is chronic injury, is the main process of training long finger force properly, excessive use and not pay attention to the protection of the reason; another is the acute injury, the finger is incorrect, incorrect technical movements cause.

\section{Reasons for the injury of the finger joints in the sport of Volleyball}

We know that sports injury is difficult to avoid, but it is necessary to reduce the movement in sports injury frequency and the extent of the damage; through the analysis of investigation and analysis, summarizes the causes for finger joint damage is mainly manifested in three aspects: one is in volleyball skills properly, two is too large, the three ball is of poor quality of its students the. In general, any movement can cause injury, volleyball is no exception, because of the characteristics of the volleyball movement determines the injury of the joint is inevitable. Therefore, it is very necessary to analyze the reasons of the injury of the finger joints in volleyball sport, which is beneficial to reduce and control the damage. 
1.There is a lack of professional preparation activities; in front of the sport it is necessary to carry out professional volleyball preparation activities, so that the joints are fully exercise. To begin at any sports are very important, to do quality, directly affects the quality of the whole sports activities and sports injury incidence; should be based on physical activity condition, especially in abnormal weather, site changes and other special circumstances. To fully do the preparatory work before the volleyball, especially the joint, this is likely to greatly reduce the probability of the injury of the finger joints.

2.Technical action is not correct. Almost all the ball moves in volleyball are the fingers to complete, including the basic skill of action of passing, serving, spiking and blocking; if the technology is improper, extremely easy to cause the incorrect hand finger injury; there are some athletes receiving a pass when the hand type error knuckle stiff, caused by finger ball the formation of finger joint damage. In addition, other parts of the body coordination is very important, such as wrist joint strength and flexibility, but also easily lead to the deformation of the technical movement of volleyball, but also cause the important cause of the injury of the joint.

3.Improper judgment of the ball. Before the movement, there is no high attention and concentration in the movement. Often appear some of the strength of the ball to determine the speed and height is not accurate. Lack of awareness, improper handling of the situation. The spike due to excessive speed, the ball can not be timely to make correct judgment to move is not in place, all of this will cause the ball when the error, resulting in finger joint damage.

\section{3 how to avoid or reduce the damage in the process of the movement of the fingers}

1.To strengthen the preventive psychological training class; to fully exploit and exert the potential of volleyball athletes, improve the effect of volleyball training. Coaches must have a purpose, consciously through a variety of targeted activities and auxiliary means. Through language, context and other tips, the athletes' psychological recognition and understanding, and the function of various parts of the body to the appropriate extension, especially for activities should conscientiously do a good job in the fingers and body; constantly improve the joint capsule and ligaments and stretch, the finger joint damage prevention in volleyball is very important the meaning of.

2.Improve technical skills training to reduce the damage in volleyball sport in time to emphasize the correctness of technical action, rationality, standardization, so that the formation of the technical action step by step. Take out the analysis of the action of the individual character, clear analysis and correct the mistakes which are easy to appear. To strengthen the body's exercise, improve the functional level, strengthen the muscles of the upper arm with the ability to hold the correct action after the standard to increase the intensity, improve the level of exercise, increase the intensity of exercise.

3.Enhance the flexibility and strength training of finger. The characteristics of volleyball athletes to volleyball athletes' physical requirements, both to action must move among force and soft stretch amplitude and can change shrinkage. Therefore, it is necessary to strengthen the training of the joint ligament of the athletes, the finger joint ligament exercises can be used: refers to the joint flexibility exercises, finger joint strength exercises.

4.To strengthen the movement of the finger joints to relax after the protection. Strengthening the protection of the fingers of the athletes is also an indispensable part of the finger joint injury. The fierce volleyball athletes after finger joints often appear different degree of strain. In this regard, we must take a different approach or the protection and treatment. If in the absence of recovery, premature training is likely to lead to exacerbations or re injured.

\section{Summary}

Therefore, in volleyball, to reduce sports injury is necessary joint, should prepare activities, proper regulation of technical movements, fully prepared, it will greatly reduce the volleyball movement refers to the movement of joint injury; at the same time to strengthen their own strength 
and flexibility training is necessary.

\section{Reference}

[1]Sun Xiaokun,Investigation and analysis of injury and prevention of volleyball[J].1999 4 [2] volleyball group. Volleyball movement [M].1999. 